\title{
Diphoton excess in phenomenological spin-2 resonance scenarios
}

\author{
Antony Martini, ${ }^{1}$ Kentarou Mawatari, ${ }^{2,3}$ and Dipan Sengupta ${ }^{2}$ \\ ${ }^{1}$ Centre for Cosmology, Particle Physics and Phenomenology (CP3), Université catholique de Louvain, \\ B-1348 Louvain-la-Neuve, Belgium \\ ${ }^{2}$ Laboratoire de Physique Subatomique et de Cosmologie, Université Grenoble-Alpes, \\ CNRS/IN2P3, 53 Avenue des Martyrs, F-38026 Grenoble, France \\ ${ }^{3}$ Theoretische Natuurkunde and IIHE/ELEM, Vrije Universiteit Brussel, and International Solvay Institutes, \\ Pleinlaan 2, B-1050 Brussels, Belgium
}

\begin{abstract}
We provide a possible explanation of a $750 \mathrm{GeV}$ diphoton excess recently reported by both the ATLAS and CMS collaborations in the context of phenomenological spin-2 resonance scenarios, where the independent effective couplings of the resonance with gluons, quarks and photons are considered. We find a parameter region where the excess can be accounted for without conflicting with dijet constraints. We also show that the kinematical distributions might help to determine the couplings to gluons and quarks.
\end{abstract}

Introduction: A heavy spin-2 resonance is one of several well-motivated new physics candidates, and provides a possible explanation of $750 \mathrm{GeV}$ diphoton excess recently reported by both the ATLAS [1] and CMS [2] collaborations with the early LHC Run-II data. The CMS analysis 2] considered the Randall-Sundrum (RS) model [3] to constrain the massive graviton as well as to explain the excess, followed by several theoretical spin-2 interpretations [4].

In this article, we interpret the excess as a spin-2 resonance that has independent effective couplings to gluons, quarks and photons as a minimal phenomenological approach. In this model, a massive spin-2 particle is produced via $g g$ and/or $q \bar{q}$ initial states and decays into a pair of photons. We explore the viable parameter region that accounts for the reported excess. The resonance can also decay into a pair of partons, giving dijet final states at the leading order, which implies that the existing 8 [11 13] and $13 \mathrm{TeV}$ [14, 15 dijet data potentially exclude a part of the model parameter space. This is also taken into account in this study. Moreover, we investigate kinematical distributions which may give more information on the couplings to gluons and quarks.

Model: We consider a massive spin-2 particle which couples to the standard model (SM) gauge and matter fields through their energy-momentum tensors [16, 17]

$$
\mathcal{L}_{\text {eff }}=-\frac{1}{\Lambda}\left[\kappa_{\gamma} T_{\mu \nu}^{\gamma}+\kappa_{g} T_{\mu \nu}^{g}+\kappa_{q} T_{\mu \nu}^{q}\right] X_{2}^{\mu \nu},
$$

where $X_{2}^{\mu \nu}$ is the spin-2 resonance and $T_{\mu \nu}^{\gamma, g, q}$ are the energy-momentum tensors; see the explicit formula, e.g. in Refs. 17, 18. Here, for simplicity, we only consider the interactions with photons, gluons and light quarks. While conventional graviton excitations have a universal coupling strength $\Lambda^{-1}$, where $\Lambda$ is the scale parameter of the theory, following [19, 20] we introduce the phenomenological coupling parameters $\kappa_{\gamma}, \kappa_{g}$ and $\kappa_{q}$ without assuming any UV models. We note that the inclusion of interactions with other SM particles such as leptons, top quarks and weak bosons is straightforward but one should then consider additional constraints from dilepton, $t \bar{t}$ and diboson searches.

We generate the signal events at the parton level by employing the Higgs Characterisation (HC) 21] model, ${ }^{1}$ where the Lagrangian (1) was implemented (based on [18, 23]). ${ }^{2}$ The HC model file is interfaced [24, 25] to the MADGraPh5_AMC@NLO event generator [26].

Analyses (rates): In the following we consider three benchmark scenarios:

$$
\begin{aligned}
& \text { I: } \kappa_{g} \neq 0, \kappa_{q}=0 \quad \text { (gluon dominant scenario), } \\
& \text { II: } \kappa_{g}=0, \kappa_{q} \neq 0 \quad \text { (quark dominant scenario), } \\
& \text { III: } \kappa_{g}, \kappa_{q} \neq 0 \quad \text { (mixed scenario), }
\end{aligned}
$$

with $\kappa_{\gamma} \neq 0$. We fix the scale $\Lambda$ at $10 \mathrm{TeV}$ throughout our study.

We start from the simple two-dimensional parameter scans, i.e. scenarios I and II. Figure 1 shows the total cross section for the spin-2 boson production at the $13 \mathrm{TeV}$ LHC (left), the diphoton branching ratio (middle), and the region accounting for the $750 \mathrm{GeV}$ diphoton excess (right) in the $\kappa_{\gamma}-\kappa_{g}$ (top) and $\kappa_{\gamma}-\kappa_{q}$ (bottom) planes.

The $X_{2}$ production rate is proportional to $\kappa_{g}^{2}\left(\kappa_{q}^{2}\right)$ in scenario I (II). For $\kappa_{g}=1\left(\kappa_{q}=1\right)$ the cross section is $4.2 \mathrm{pb}(0.6 \mathrm{pb})$, where the NNPDF2.3 [27] is employed and the factorisation scale is fixed at $m_{X_{2}}=750 \mathrm{GeV}$. For $\kappa_{g} \sim \kappa_{q}$ the production rate of the gluon-induced process is larger than that of the quark-induced one by a factor of seven due to the larger parton luminosity.

The diphoton branching ratio depends on both $\kappa_{\gamma}$ and $\kappa_{g, q}$, and is determined by the partial widths

$$
\Gamma_{\{\gamma \gamma, g g, q q\}}=\frac{m_{X_{2}}^{3}}{80 \pi \Lambda^{2}}\left\{\kappa_{\gamma}^{2}, 8 \kappa_{g}^{2}, 6 \kappa_{q}^{2}\right\},
$$

\footnotetext{
1 The model file is publicly available at the FEYnRules $[22]$ repository.

2 Although the HC model is designed to study the spin-parity nature of the $125 \mathrm{GeV}$ Higgs boson, one can easily change its mass $m_{X_{2}}$ as a parameter.
} 

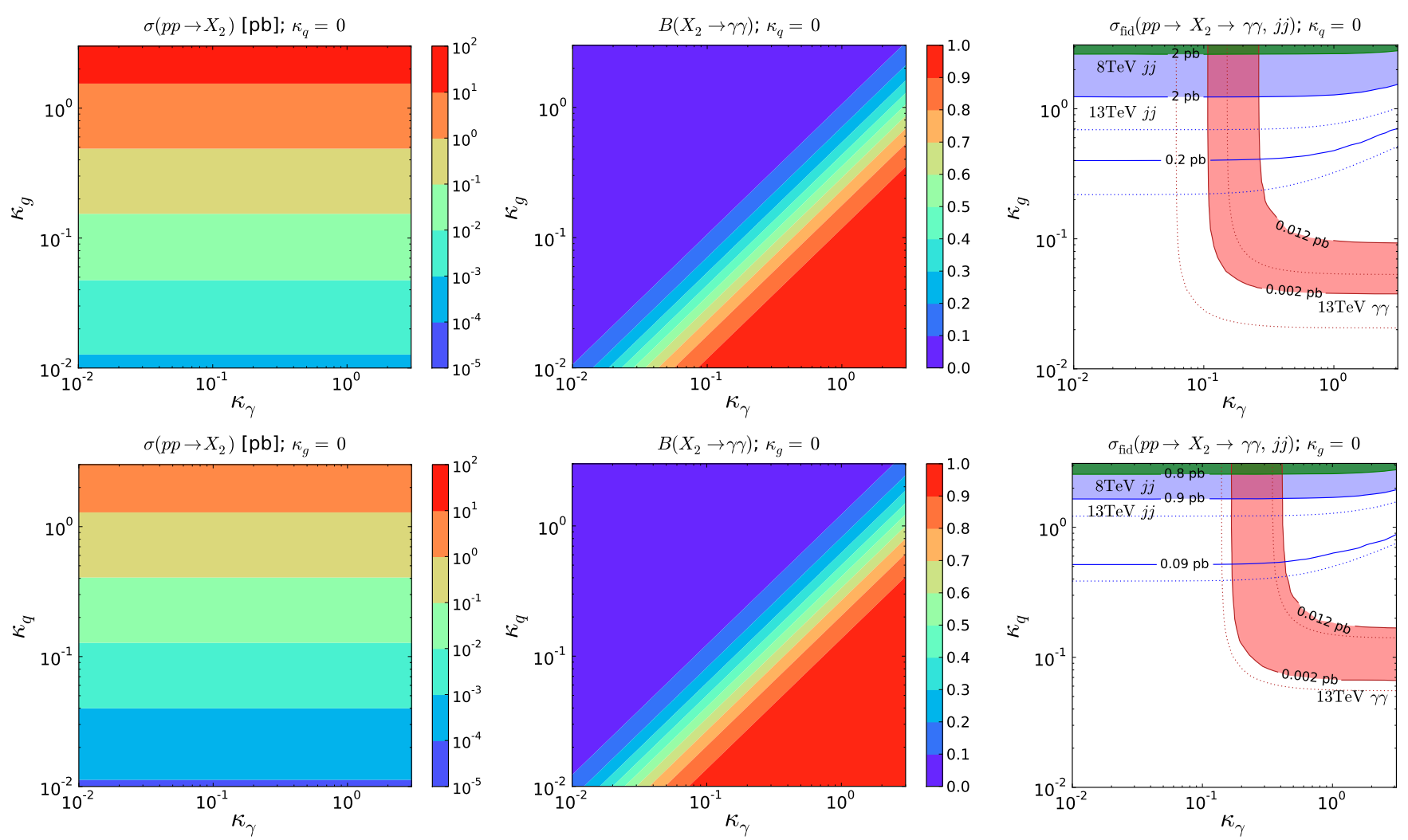

FIG. 1: Left: Total cross section for the spin-2 boson production at the $13 \mathrm{TeV}$ LHC. Center: Diphoton branching ratio. Right: Regions explaining the $750 \mathrm{GeV}$ diphoton excess and constrained by the dijet data; see the text for details. The gluon (quark) dominant scenario I (II) is shown in the top (bottom) panels.

where four quark flavours are considered. MADWIDTh [28] provides the above partial decay rates for each parameter point. The $X_{2}$ total width is 1.4 (1.1) $\mathrm{GeV}$ for $\kappa_{\gamma}=\kappa_{g(q)}=3$; i.e. the $X_{2}$ has a narrow width for the entire parameter space in our scenarios. We will briefly discuss the broad resonance scenario in the summary section.

In the right plots in Fig. 1 the red shaded region can fit the ATLAS and CMS diphoton excess (2-12 fb) 1, 2, where, in addition to the minimal cuts $p_{T}^{\gamma}>25 \mathrm{GeV}$ and $\left|\eta^{\gamma}\right|<2.37$, the fiducial cut [1]

$$
p_{T}^{\gamma}>300 \mathrm{GeV}
$$

is imposed. The red dotted lines refer to the same region but without any cuts, i.e. derived by the simple $\sigma\left(p p \rightarrow X_{2}\right) \times B\left(X_{2} \rightarrow \gamma \gamma\right)$ calculation. One can easily understand the shape of the region from (the left plot) $\times$ (the middle plot). Analytically, $\sigma \times B_{\gamma \gamma} \propto \kappa_{\gamma}^{2}$ for $\kappa_{\gamma} \ll \kappa_{g, q}$, while $\sigma \times B_{\gamma \gamma} \propto \kappa_{g, q}^{2}$ for $\kappa_{\gamma} \gg \kappa_{g, q}$. For the quantitative estimation, on the other hand, we find that the effect of the fiducial cut is significant. We checked that the effect of the minimal cuts is small. It should be noted that the effect of the fiducial cut is larger for the gluon case (I) than that for the quark one (II). This can be explained by the difference of the kinematical distributions between the two cases, which will be discussed later.

As mentioned in the Introduction, the existing dijet data may constrain the allowed parameter space. The green and blue shaded regions in the right plots of Fig. 1 present the exclusion by the dijet analyses at $\sqrt{s}=8$ [1113] and $13 \mathrm{TeV}$ [14, 15], respectively. To evaluate the dijet constraints, in addition to the minimal cuts $p_{T}^{j}>$ $30 \mathrm{GeV}$ and $\left|\eta^{j}\right|<2.5$, the selection cut [13, 14]

$$
\left|\Delta \eta^{j j}\right|<1.3
$$

is imposed. We take the $95 \%$ confidence level (CL) upper limits on the cross section at $\sqrt{s}=8 \mathrm{TeV}$ from the CMS analysis [13: $2.0(0.8)$ pb for the $g g(q \bar{q})$ type dijet resonance. For the $13 \mathrm{TeV}$ dijet constraint, on the other hand, there is no analysis below $m_{j j}<1 \mathrm{TeV}$ yet [14, 15], and hence we simply extrapolate the CMS limits at the $1.5 \mathrm{TeV}$ resonance mass, i.e. $2.0(0.9) \mathrm{pb}$ for the $g g(q \bar{q})$ type resonance [14, as a conservative constraint. We also show the ten-time stronger limits $(0.2 \mathrm{pb}$ for $g g$ and $0.09 \mathrm{pb}$ for $q \bar{q})$ at $\sqrt{s}=13 \mathrm{TeV}$ as a future constraint. Similar to the diphoton case, the blue dotted lines show the $13 \mathrm{TeV}$ dijet constraints derived by $\sigma \times B_{j j}$ without any cuts. For the dijet case, $\sigma \times B_{j j} \propto \kappa_{g, q}^{2}$ for $\kappa_{\gamma} \ll \kappa_{g, q}$, while $\sigma \times B_{j j} \propto \kappa_{g, q}^{4} / \kappa_{\gamma}^{2}$ for $\kappa_{\gamma} \gg \kappa_{g, q}$.

We conclude that the current (and near future) dijet constraints are not strong enough to exclude the 


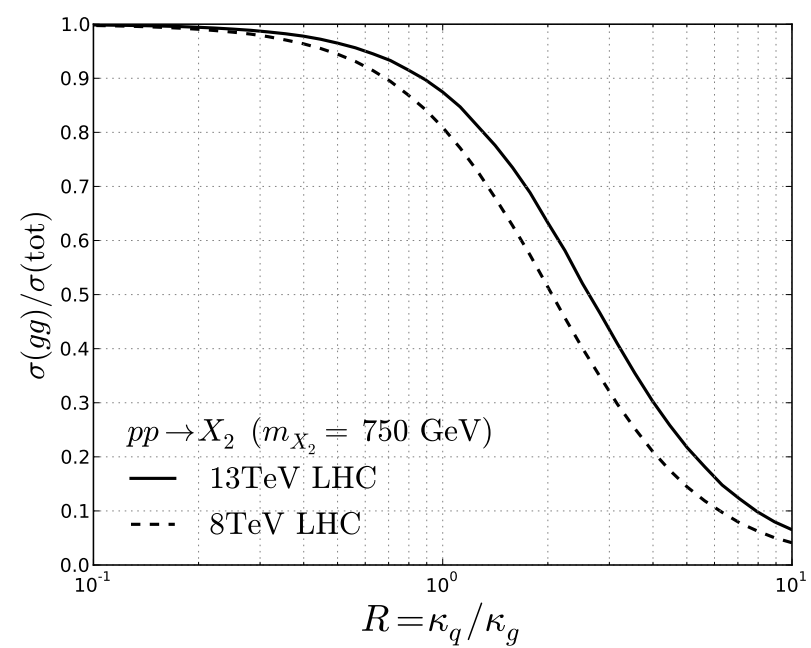

FIG. 2: Contributions of $g g$ and $q \bar{q}$ subprocesses to $750 \mathrm{GeV}$ spin-2 particle production at the LHC as a function of $R=$ $\kappa_{q} / \kappa_{g}$.

parameter region accounting for the diphoton excess for both the $g g$ and $q \bar{q}$ scenarios. We note that the $\kappa_{g(q)}=\kappa_{\gamma}=0.25(0.4)$ case gives $\sigma_{\text {fid }}(\gamma \gamma) \sim 10 \mathrm{fb}$. Since the $\kappa_{g}-\kappa_{q}$ mixed scenario III is estimated by the linear combination of the two scenarios I and II, we can always adjust the parameters to fit the diphoton excess without conflicting the dijet constraints. However, the information of the total rate is not enough to determine a unique $\left(\kappa_{g}, \kappa_{q}\right)$ solution. We note that since the effect of the fiducial cuts is sizeable, a more precise analysis should include, e.g., next-to-leading-order (NLO) corrections in QCD [29], effects of additional partons in the final state [21, hadronisation, and detector response. Such a study will be reported elsewhere.

Analyses (distributions): We now turn to the possibility of determining the values of $\kappa_{g}$ and $\kappa_{q}$ for the mixed scenario III in the diphoton events. Since angular dependence at the partonic centre-of-mass frame for a spin-2 particle production is different between $g g$ and $q \bar{q}$ initial states 30, kinematical distributions may be able to provide additional information.

Figure 2 shows the individual contributions of $g g$ and $q \bar{q}$ initial states in $p p \rightarrow X_{2}$ production at the $13 \mathrm{TeV}$ LHC as a function of the ratio between $\kappa_{q}$ and $\kappa_{g}$, $R \equiv \kappa_{q} / \kappa_{g}$. As a reference, the $8 \mathrm{TeV}$ LHC case is also shown by a dashed line. As mentioned above, the gluon-induced process is dominant at $R=1$, i.e. the universal coupling case. To present the kinematical distributions below, we choose four benchmark points as $R=0.1,1.0,2.5,10$, which give $99 \%, 87 \%, 52 \%, 7 \% g g$ contributions, respectively. For a certain $R$, we can always find the $\kappa$ parameters to satisfy both the excess and the constraints.

Figure 3 shows the normalised $\eta$ and $p_{T}$ distributions of the photon for the above benchmark points.
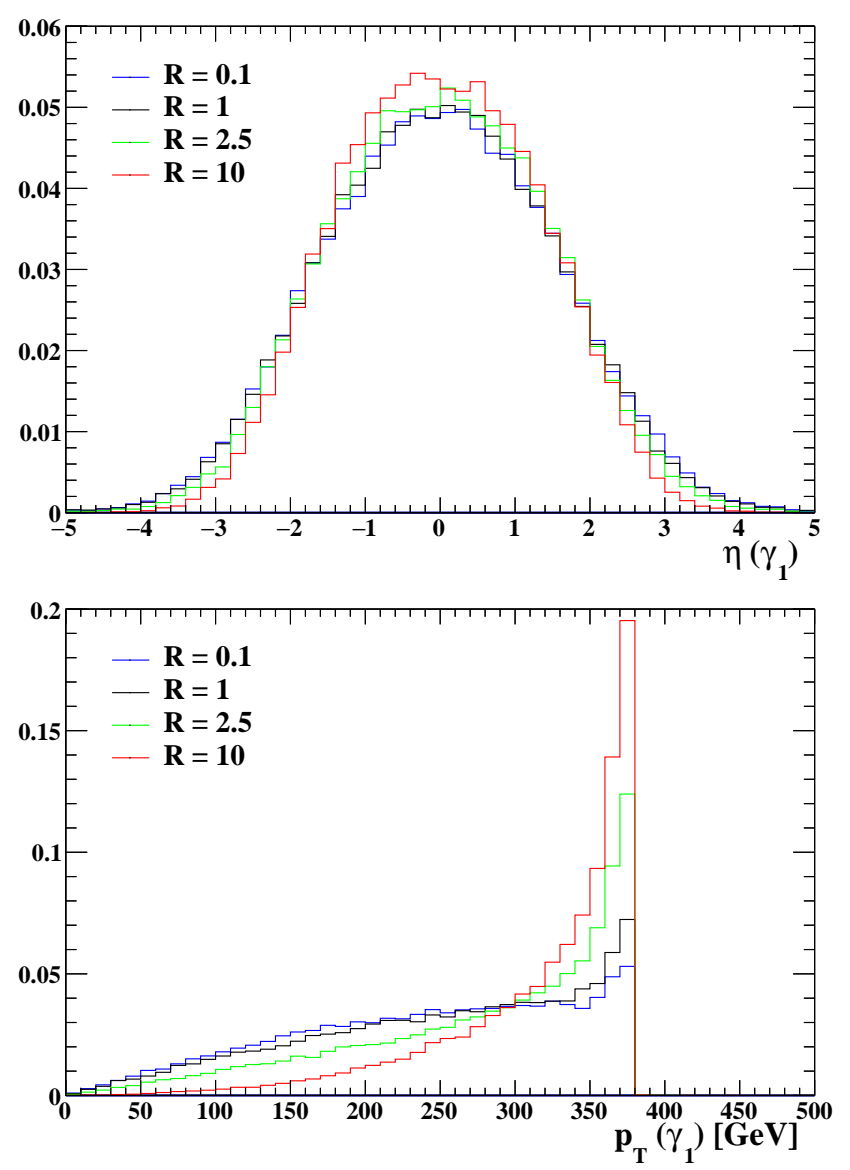

FIG. 3: Normalised photon pseudorapidity (top) and transverse momentum (bottom) distributions in $p p \rightarrow X_{2} \rightarrow \gamma \gamma$ process at $\sqrt{s}=13 \mathrm{TeV}$ for different $R$ values, without any kinematical cuts.

Comparing with the gluon-induced process $(R=0.1)$, $q \bar{q}$ annihilation $(R=10)$ produces the photons in the more central and higher- $p_{T}$ regions. These kinematical features can explain the large difference of the signal acceptance between scenarios I and II, observed in Fig. 1 (right). Although the fiducial cut diminishes the distinction between the different mixed cases, we could still observe the difference once we have enough data. A variation of the fiducial volumes can help to resolve the $\kappa_{g}$ and $\kappa_{q}$ values. One caveat is that nonuniversal couplings to gluons and quarks, $\kappa_{g} \neq \kappa_{q}$, give rise to a unitarity-violating behaviour at the NLO in QCD, especially in the $p_{T}^{X_{2}}>m_{X_{2}}$ region [21. We note, however, that we can always find the region explaining the excess under the $\kappa_{g}=\kappa_{q}$ condition; e.g. $\kappa_{g}=\kappa_{q}=0.1$ with $\kappa_{\gamma}=0.4$, providing $\sigma_{\text {fid }}(\gamma \gamma) \sim 10 \mathrm{fb}$.

Summary and discussion: We interpreted the $750 \mathrm{GeV}$ diphoton anomaly recently reported at the LHC as a spin-2 narrow resonance which only couples to photons, gluons and light quarks. We introduced the three independent coupling parameters, $\kappa_{\gamma}, \kappa_{g}$ and $\kappa_{q}$, and found 
the viable parameter regions explaining the diphoton excess without conflicting the dijet constraints. The diphoton kinematical distributions are distinctive between the $g g$ and $q \bar{q}$ subprocesses, which leads to different signal acceptances and provides a possibility to determine the parameters uniquely.

Although we only considered the spin-2 couplings to the SM particles relevant to the diphoton and dijet analyses as a minimal phenomenological framework without assuming any UV models, extensions of the model are straightforward, but should include more constraints such as dilepton, diboson, and $t \bar{t}$ final states.

Before closing, we mention the broader resonance case, which is indicated by the ATLAS data [1]. Our viable parameter region only provides a narrow width even after including all the SM decay channels. In passing, $\Gamma_{X_{2}} \sim 45 \mathrm{GeV}$ requires $\Lambda \sim 950 \mathrm{GeV}$ in the RS model with a universal coupling, which is excluded by the dijet data. Therefore, other decay channels such as dark matter [7] are necessary to have such a wider resonance.

Acknowledgements: We would like to thank Sabine Kraml and Fabio Maltoni for valuable comments on the manuscript.

The work of A.M. is supported by the IISN "MadGraph" convention 4.4511.10 and the IISN "Fundamental interactions" convention 4.4517.08. The work of K. M. is supported by the Theory-LHC-France initiative of the CNRS (INP/IN2P3). The work of D.S. is supported by the French ANR project DMAstroLHC, ANR-12-BS050006.
[1] ATLAS (2015), ATLAS-CONF-2015-081.

[2] CMS (2015), CMS-PAS-EXO-15-004.

[3] L. Randall and R. Sundrum, Phys. Rev. Lett. 83, 3370 (1999), hep-ph/9905221.

[4] R. Franceschini, G. F. Giudice, J. F. Kamenik, M. McCullough, A. Pomarol, R. Rattazzi, M. Redi, F. Riva, A. Strumia, and R. Torre (2015), 1512.04933.

[5] M. Low, A. Tesi, and L.-T. Wang, JHEP 03, 108 (2016), 1512.05328 .

[6] M. T. Arun and P. Saha (2015), 1512.06335.

[7] C. Han, H. M. Lee, M. Park, and V. Sanz, Phys. Lett. B755, 371 (2016), 1512.06376.

[8] J. S. Kim, K. Rolbiecki, and R. R. de Austri (2015), 1512.06797.

[9] C. Csaki, J. Hubisz, S. Lombardo, and J. Terning (2016), 1601.00638.

[10] M. R. Buckley (2016), 1601.04751.

[11] S. Chatrchyan et al. (CMS), Phys. Rev. D87, 114015 (2013), 1302.4794.

[12] G. Aad et al. (ATLAS), Phys. Rev. D91, 052007 (2015), 1407.1376.

[13] CMS (2015), CMS-PAS-EXO-14-005.

[14] V. Khachatryan et al. (CMS), Phys. Rev. Lett. 116, 071801 (2016), 1512.01224.

[15] G. Aad et al. (ATLAS), Phys. Lett. B754, 302 (2016), 1512.01530 .

[16] G. F. Giudice, R. Rattazzi, and J. D. Wells, Nucl. Phys. B544, 3 (1999), hep-ph/9811291.

[17] T. Han, J. D. Lykken, and R.-J. Zhang, Phys. Rev. D59, 105006 (1999), hep-ph/9811350.

[18] K. Hagiwara, J. Kanzaki, Q. Li, and K. Mawatari, Eur.
Phys. J. C56, 435 (2008), 0805.2554.

[19] J. Ellis, R. Fok, D. S. Hwang, V. Sanz, and T. You, Eur. Phys. J. C73, 2488 (2013), 1210.5229.

[20] C. Englert, D. Goncalves-Netto, K. Mawatari, and T. Plehn, JHEP 01, 148 (2013), 1212.0843.

[21] P. Artoisenet et al., JHEP 11, 043 (2013), 1306.6464.

[22] A. Alloul, N. D. Christensen, C. Degrande, C. Duhr, and B. Fuks, Comput.Phys.Commun. 185, 2250 (2014), 1310.1921.

[23] P. de Aquino, K. Hagiwara, Q. Li, and F. Maltoni, JHEP 06, 132 (2011), 1101.5499.

[24] C. Degrande, C. Duhr, B. Fuks, D. Grellscheid, O. Mattelaer, and T. Reiter, Comput. Phys. Commun. 183, 1201 (2012), 1108.2040.

[25] P. de Aquino, W. Link, F. Maltoni, O. Mattelaer, and T. Stelzer, Comput. Phys. Commun. 183, 2254 (2012), 1108.2041

[26] J. Alwall, R. Frederix, S. Frixione, V. Hirschi, F. Maltoni, O. Mattelaer, H. S. Shao, T. Stelzer, P. Torrielli, and M. Zaro, JHEP 07, 079 (2014), 1405.0301.

[27] R. D. Ball et al., Nucl. Phys. B867, 244 (2013), 1207.1303.

[28] J. Alwall, C. Duhr, B. Fuks, O. Mattelaer, D. G. zturk, and C.-H. Shen, Comput. Phys. Commun. 197, 312 (2015), 1402.1178.

[29] M. C. Kumar, P. Mathews, V. Ravindran, and A. Tripathi, Nucl. Phys. B818, 28 (2009), 0902.4894.

[30] B. C. Allanach, K. Odagiri, M. J. Palmer, M. A. Parker, A. Sabetfakhri, and B. R. Webber, JHEP 12, 039 (2002), hep-ph/0211205. 